\title{
Australian doctors' satisfaction with their work: results from the MABEL longitudinal survey of doctors
}

\author{
Catherine M Joyce, Stefanie Schurer, Anthony Scott, John Humphreys and Guyonne Kalb
}

$\longrightarrow$ octors' job satisfaction levels play an important role in their decisions to reduce working hours or leave the medical workforce, influencing doctor shortages and patient access to care. ${ }^{1-3}$ Doctors' job satisfaction is also associated with patient satisfaction and quality of care, through factors such as continuity of care and prescribing patterns. ${ }^{4}$

Most studies of job satisfaction among Australian doctors focus on general practitioners and show high levels of job satisfaction among small samples. ${ }^{5-8}$ Female GPs and GPs working in rural areas often report higher levels of satisfaction than other GPs, with similar findings being reported internationally. ${ }^{9}$ Australian anaesthetists are reported to have high levels of job satisfaction, ${ }^{10}$ as are ophthalmologists, ${ }^{11}$ hospital consultants ${ }^{12}$ and most intensivists, although the latter also report very high levels of burnout. ${ }^{13}$ For GPs and Australian emergency physicians, dissatisfaction is associated with their workload, administrative demands, working hours (ie, shift work, night shifts and on-call work) and lack of recognition. ${ }^{5-9,14}$

Although specialists are thought to be more satisfied than GPs, this has not been tested. International research indicates that job satisfaction varies by practice type and specialty. ${ }^{15}$ Whether such differences reflect the characteristics of the specialties or the characteristics of the doctors who self-select into these specialties is important when designing policies to increase job satisfaction.

The aim of our study was to investigate whether levels of job satisfaction and determinants of satisfaction differ between four groups of doctors in the Australian medical workforce: GPs, specialists, specialists-intraining and hospital non-specialists.

\section{METHODS}

\section{Participants}

Data from the baseline cohort of the Medicine in Australia - Balancing Employment and Life (MABEL) longitudinal survey of doctors were collected between June and November 2008. ${ }^{16}$ All 54750 doctors undertaking some clinical work listed in the Medical Directory of Australia ${ }^{17}$ were invited

\section{ABSTRACT}

Objective: To compare the level and determinants of job satisfaction between four groups of Australian doctors: general practitioners, specialists, specialists-in-training, and hospital non-specialists.

Design, participants and setting: National cross-sectional questionnaire survey as part of the baseline cohort of a longitudinal survey of Australian doctors in clinical practice (Medicine in Australia - Balancing Employment and Life [MABEL]), undertaken between June and November 2008, including 5193 Australian doctors (2223 GPs, 2011 specialists, 351 hospital non-specialists, and 608 specialists-in-training).

Main outcome measures: Job satisfaction scores for each group of doctors; the association between job satisfaction and doctor, job and geographical characteristics. Results: $85.7 \%$ of doctors were moderately or very satisfied with their jobs. There were no differences in job satisfaction between GPs, specialists and specialists-in-training. Hospital non-specialists were the least satisfied compared with GPs (odds ratio [OR], 0.56 [95\% Cl, 0.39-0.81]). For all doctors, factors associated with high job satisfaction were a good support network (OR, 1.72 [95\% Cl, 1.41-2.10]), patients not having unrealistic expectations $(\mathrm{OR}, 1.48$ [95\% Cl, 1.25-1.75]), and having no difficulty in taking time off work (OR, $1.48[95 \% \mathrm{Cl}, 1.20-1.84])$. These associations did not vary across doctor types. Compared with GPs, on-call work was associated with lower job satisfaction for specialists (OR, 0.48 [95\% Cl, 0.23-0.98]) and hospital non-specialists $(\mathrm{OR}, 0.25[95 \% \mathrm{Cl}$, $0.08-0.83])$.

Conclusion: This is the first national survey of job satisfaction for doctors in Australia. It provides an important baseline to examine the impact of future health care reforms and other policy changes on the job satisfaction of doctors.

MJA 2011; 194: 30-33

by mail to participate. Doctors could choose to complete a paper copy of the questionnaire or an online version through the secure study website. Doctors in remote areas were given an honorarium to maximise their response rates. Four categories of doctors were used: ${ }^{18}$ GPs and GP registrars, specialists, specialists-in-training and hospital non-specialists (interns, postgraduate non-specialists in years 1 to 3 of their training and hospital medical officers).

The study was approved by the University of Melbourne Faculty of Economics and Commerce Human Ethics Advisory Group and the Monash University Standing Committee on Ethics in Research Involving Humans.

\section{Variables used}

Job satisfaction was measured with the widely used 10-item version of the WarrCook-Wall job content questionnaire, which includes nine questions about aspects of a job and one global satisfaction question. ${ }^{9,19,20}$ Participants responded on a five- point Likert scale from "very dissatisfied" (0) to "very satisfied" (4).

The MABEL baseline questionnaire collected detailed data on working arrangements previously shown to be associated with job satisfaction for doctors. These included hours worked, professional relationships and support, on-call work, time pressures, patient characteristics, age, sex, family circumstances and geographical location.

\section{Analysis}

As most doctors were moderately or very satisfied (Box 1), overall job satisfaction was coded as a binary dependent variable equal to 1 if the doctor was "very satisfied" and 0 otherwise. This approach yielded the best goodness-of-fit when compared with coding "moderately satisfied" and "very satisfied" equal to 1 . Using a continuous or ordinal dependent variable made little difference to the results. Using Stata, version 10.1 (StataCorp, College Station, Tex, USA), a logistic regression model was estimated, including doctor type as a categorical explanatory 
1 Job satisfaction by doctor type*

\begin{tabular}{|c|c|c|c|c|c|}
\hline & General practitioners & Specialists & Hospital non-specialists & Specialists-in-training & All doctors \\
\hline $\begin{array}{l}\text { Mean job satisfaction } \\
\text { (range, } 0-4)(95 \% \mathrm{Cl})\end{array}$ & $3.06(3.02-3.11)$ & $3.15(3.10-3.19)$ & $2.85(2.76-2.94)$ & $2.96(2.89-3.03)$ & $3.06(3.04-3.10)$ \\
\hline \multicolumn{6}{|l|}{ Distribution (\%) $(95 \% \mathrm{Cl})$} \\
\hline Very dissatisfied & $1.56(0.97-2.14)$ & $1.10(0.61-1.58)$ & $0.70(-0.10-1.49)$ & $1.59(0.49-2.70)$ & $1.34(0.99-1.70)$ \\
\hline Moderately dissatisfied & $8.54(7.30-9.78)$ & $7.98(6.72-9.24)$ & $11.93(8.34-15.53)$ & $7.27(5.06-9.49)$ & $8.45(7.64-9.26)$ \\
\hline Not sure & $4.54(3.60-5.48)$ & $3.65(2.78-4.51)$ & $6.03(3.40-8.66)$ & $6.15(4.11-8.20)$ & $4.55(3.94-5.16)$ \\
\hline Moderately satisfied & $52.71(50.49-54.93)$ & $49.78(47.50-52.06)$ & $64.41(59.13-69.70)$ & $63.59(59.42-67.75)$ & $53.92(52.48-55.37)$ \\
\hline Very satisfied & $32.65(30.57-34.73)$ & $37.49(35.28-39.71)$ & $16.93(13.00-20.96)$ & $21.39(17.81-24.97)$ & $31.74(30.41-33.07)$ \\
\hline Unweighted sample size $^{\dagger}$ & 2223 & 2011 & 351 & 608 & 5193 \\
\hline
\end{tabular}

variable to test the hypothesis that job satisfaction was different between doctor types. Correlations between independent variables were checked for multicollinearity, and the functional form of continuous variables was decided on the basis of the Pregibon link test. Goodness-of-fit tests were conducted using the Hosmer-Lemeshow test and the Akaike and Bayesian information criteria. Wald tests of joint significance for each block of coefficients relating to a categorical or continuous variable were used to assess whether the variable was associated with job satisfaction.

Survey weights were used to adjust for survey response bias with respect to age, sex, doctor type, geographical location, state and whether they received an honorarium. ${ }^{21}$ The precision of the estimated strength of relationship between the independent and dependent variables was robust when either the survey weights or observations with high leverage (ie, influential observations, such as outliers) were excluded from the analysis. A model including interaction terms between doctor type and each independent variable was estimated to test hypotheses about whether the factors associated with job satisfaction were different for each type of doctor. ${ }^{22}$

\section{RESULTS}

A total of 10498 doctors (19.4\% of those contacted and eligible) responded. The highest response rate was from specialists (4310; 22.3\%), followed by specialists-intraining $(864 ; 20.6 \%)$, GPs (3873; 17.7\%) and hospital non-specialists ( $1451 ; 16.5 \%)$. Online responses were received from $30.4 \%$ of all respondents (25.4\% of GPs, $27.6 \%$ of specialists, $47.6 \%$ of hospital non-specialists and $38 \%$ of specialists-in-training). Respondents showed small but statistically significant differences from the population of doctors listed in the Medical Directory of Australia list with respect to age, sex, doctor type and geographical location. ${ }^{16}$ Results presented are based on the 5193 respondents who provided complete data for all variables in the regression model (2223 GPs, 2011 specialists, 351 hospital non-specialists and 608 specialists-in-training).

Most doctors were either moderately satisfied or very satisfied with their jobs (Box 1 ). Specialists were most likely to be very satisfied (37.5\%), followed by GPs (32.7\%), specialists-in-training (21.4\%) and hospital non-specialists (16.9\%). Fewer than $2 \%$ of doctors in each group were very dissatisfied, but $12 \%$ of hospital non-specialists were moderately dissatisfied, compared with 7\%$8 \%$ for other doctors.

Descriptive statistics for independent variables in the regression model are available from the authors on request. After controlling for doctor, job and geographical characteristics, specialists and specialists-intraining were just as likely to report high job satisfaction as GPs, but the odds of hospital non-specialists being very satisfied were 0.56 times that of GPs (Box 2).

There was no significant difference (at the $5 \%$ level) between the proportions of male and female doctors who reported being very satisfied with their job (Box 2). The age and age-squared variables show that the odds of reporting high job satisfaction follow a Ushape, such that satisfaction falls with age at first, but slowly flattens out and then rises again as doctors approach retirement. The odds of being very satisfied were lower for doctors with lower levels of self-assessed health. Doctors with higher household incomes were more likely to be satisfied.

Other factors associated with being very satisfied were having a good professional support network (odds ratio [OR], 1.72 [95\% CI, 1.41-2.10] compared with neutrality) and ease of taking time off work (OR,
1.48 [95\% CI, 1.20-1.84] compared with neutrality) (Box 2).

Doctors who agreed with the statement "My patients have unrealistic expectations about how I can help them" were less likely to be very satisfied than those who were neutral on this statement (OR, 0.52 [95\% CI, 0.43-0.62]), while doctors who disagreed were more likely to be very satisfied (OR, 1.48 [95\% CI, 1.25-1.75]) (Box 2).

Doctors with friends and family in their current work location were more likely to be very satisfied with their job than those who were neutral on this statement (OR, 1.57 [95\% CI, 1.24-2.00]). Compared with doctors in New South Wales, doctors practising in the Australian Capital Territory, Queensland and Western Australia were between 1.2 and 1.7 times more likely to be very satisfied.

The odds ratios were statistically significant for only two interaction terms. For specialists who had a child and a partner, the odds of being very satisfied were greater than those of a GP with a child and a partner (OR, 2.05 [95\% CI, 1.28-3.29]) (data not shown). Specialists who did on-call work were less likely to report being very satisfied than GPs doing on-call work (OR, 0.48 [95\% CI, 0.23-0.98]). There were similar findings for hospital non-specialists who did on-call work compared with GPs (OR, 0.25 [95\% CI, 0.08-0.83]) (data not shown).

\section{DISCUSSION}

Most doctors in Australia are moderately or very satisfied with their jobs, with no significant differences in job satisfaction between GPs, specialists and specialists-in-training. Hospital non-specialists appeared to be less satisfied than GPs. Interns and hospital medical officers comprise $85 \%$ of hospital non-specialists, and are likely to experience the least autonomy over their work and also to work the longest hours, factors previously 
2 Association between doctors being "very satisfied" in their job and doctor, job and geographical characteristics: results from logistic regression*

\section{Type of doctor (reference: GP)}

Specialist

Hospital non-specialist

Specialist-in-training

Female (reference: male)

Age

Age square

Family status (reference: single)

Has partner, no children

Has children, no partner

Has partner and children

Self-assessed health (reference: excellent)

Very good

Satisfactory

Bad or poor

Annual household income ${ }^{\S}$

Poor support network of similar doctors (reference: neutral)

Agree

Disagree

Difficult to take time off work (reference: neutral)

Agree

Disagree

Hours worked per week (reference: 35-54)

$\leqslant 35$

55-59

$\geqslant 60$

Doing own on-call work (yes $=1$ )

Degree obtained outside Australia (yes $=1$ )

Patients have unrealistic expectations (reference: neutral)

Agree

Disagree

Few friends and family locally (reference: neutral)

Agree

Disagree

ASGC remoteness area (reference: major city)

Inner regional

Outer regional, remote and very remote

State (reference: New South Wales)

Australian Capital Territory

Northern Territory

Queensland

South Australia

Tasmania

Victoria
Western Australia

$$
\mathrm{OR}^{\dagger}(95 \% \mathrm{Cl})(n=5193)
$$

$P^{\ddagger}$

0.022

$0.99(0.84-1.18)$

$0.56(0.39-0.81)$

$0.82(0.60-1.12)$

$1.16(0.98-1.37)$

0.078

$0.92(0.87-0.98)$

0.004

$1.01(1.01-1.02)$

$<0.001$

0.264

$1.13(0.86-1.49)$

$1.48(0.97-2.24)$

$1.24(0.95-1.62)$

$0.67(0.57-0.79)$

$0.47(0.39-0.58)$

$0.30(0.21-0.42)$

$1.36(1.18-1.56)$

$<0.001$

$<0.001$

$0.93(0.74-1.17)$

$1.72(1.41-2.10)$

$0.83(0.66-1.03)$

$1.48(1.20-1.84)$

$1.08(0.90-1.30)$

$0.78(0.59-1.05)$

$0.91(0.72-1.14)$

$0.88(0.75-1.04)$

0.133

$0.89(0.60-1.32)$

0.555

$<0.001$

$0.52(0.43-0.62)$

$1.48(1.25-1.75)$

$1.16(0.89-1.52)$

$1.57(1.24-2.00)$

$1.22(0.99-1.51)$

$1.22(0.90-1.65)$

$1.71(1.06-2.74)$

$1.17(0.59-2.32)$

$1.24(1.00-1.53)$

$1.28(0.98-1.69)$

$0.89(0.58-1.37)$

$1.18(0.99-1.41)$

$1.53(1.18-1.98)$
$\mathrm{OR}=$ odds ratio. $\mathrm{ASGC}=$ Australian Standard Geographical Classification. ${ }^{*}$ Data adjusted with survey weights. $†$ ORs indicate differences between each group within a categorical variable, compared with designated reference group. $¥ P$ indicates strength of association between each variable and job satisfaction. $\S$ Equivalised household income refers to annual household income divided by the square root of the number of household members; log transformation of household income was chosen due to better model fit.

Pseudo-log likelihood $=-2748$.
3 Key factors associated with high job satisfaction

Job characteristics

- Realistic patient expectations

- Good professional support networks

- Being able to take time off

Geographical factors

- Friends and family in current work location

- Working outside New South Wales

Doctor characteristics

- Being younger or close to retirement

- Good self-reported health

- High household income

found to be associated with lower job satisfaction. $^{5-9,14}$

The key drivers of overall job satisfaction demonstrated by our study are summarised in Box 3. While we can identify associations, the direction of causality in these relationships is unknown.

Agreeing that their patients had realistic expectations was associated with high job satisfaction, consistent with previous studies examining job satisfaction and "difficult encounters". ${ }^{23,24}$ Previous research has shown that reporting of "heartsink" patients (difficult or problem patients, about whom doctors tend to feel helpless) was associated with a higher perceived workload, a lack of training in counselling and communication skills, and a lack of appropriate postgraduate qualifications, rather than with patient characteristics. ${ }^{24}$ It may be that a high number of difficult encounters leads to lower job satisfaction, or that dissatisfied doctors perceive a higher number of encounters as difficult.

Having professional support was strongly associated with job satisfaction, consistent with research showing that it is a key factor in retention for rural areas, and that graduates' perceptions of professional support influence their career choices. ${ }^{25-29}$ The associations between location of work and job satisfaction may reflect factors such as the availability of health resources, population factors, variation in working conditions, or the cost of living.

Whether a doctor had qualified overseas was not associated with job satisfaction, suggesting that overseas-trained doctors have the same level of satisfaction as Australian-trained doctors.

There was no association between job satisfaction and remoteness, although this may be an artefact of the Australian Standard Geographical Classification remoteness areas classification, which groups hetero- 
geneous locations within each category. We tested for interactions between remoteness and whether an individual obtained a degree outside Australia, as one might expect having an overseas qualification to have more of an effect in a rural area, but these were jointly insignificant $(P=0.71)$.

Being on-call and the number of hours worked per week were not statistically significant in the overall model and did not differ across doctor types, although specialists doing on-call work were less likely to be satisfied than GPs doing on-call work. With regard to hours worked, this is surprising, given that results from previous research show some association, especially for those who are working very long hours and experience burnout. ${ }^{9}$ However, the culture of clinical practice is that working long hours is the norm and is necessary to complete training programs. Hence, a high reported job satisfaction for doctors working long hours may reflect the fact that actual hours worked are in line with expectations.

The data used in our analysis were selfreported and from a self-selected group of doctors. The generalisability of the findings is improved by the use of survey weights in the analysis. The representativeness of the data has been discussed in detail elsewhere. ${ }^{16}$

As large numbers of medical graduates begin to enter the health care system, the finding that hospital non-specialists experience the lowest job satisfaction is a cause for concern. This may be part of the medical training culture, and therefore may be accepted by many as not being a problem. However, an increase in the number of junior doctors combined with a fixed pool of doctors to supervise their training is unlikely to improve the situation. It will be important to monitor job satisfaction and conduct further research into burnout and exits from the profession by junior doctors.

The associations reported here provide an important baseline for examining changes in job satisfaction over time in response to health policy reform and changes in job characteristics. Questions about whether job satisfaction influences doctors' decisions about working hours, retention and mobility are central to examining patterns of access to health care, and require further research.

\section{ACKNOWLEDGEMENTS}

This work was supported by a National Health and Medical Research Council Health Services research grant and the Australian Department of Health and Ageing. The views in this paper are those of the authors alone. We thank the doctors who gave their valuable time to participate in the Medicine in
Australia - Balancing Employment and Life Study; Anne Leahy, Sung-Hee Jeon, Julia Witt, Terence Cheng, Daniel Kuehnle, Matthew McGrail, Michelle Mclsaac, Durga Shrestha, Danny Hills and Peter Sivey for data cleaning and comments; and Rory Wolfe for biostatistical advice.

\section{COMPETING INTERESTS}

None identified.

\section{AUTHOR DETAILS}

Catherine M Joyce, BA(Hons), MPsych, PhD, Senior Lecturer, Department of Epidemiology and Preventive Medicine ${ }^{1}$

Stefanie Schurer, MSc, PhD, Research Fellow ${ }^{2}$ Anthony Scott, BA, MSc, PhD, Professorial Research Fellow ${ }^{2}$

John Humphreys, BA(Hons), DipEd, PhD,

Professor of Rural Health Research, School of Rural Health ${ }^{1}$

Guyonne Kalb, MEc, PhD, Principal Research Fellow ${ }^{2}$

1 Monash University, Melbourne, VIC.

2 Melbourne Institute of Applied Economic and Social Research, University of Melbourne,

Melbourne, VIC.

Correspondence:

catherine.joyce@monash.edu

\section{REFERENCES}

1 Landon BE, Reschovsky JD, Pham HH, Blumenthal $D$. Leaving medicine: the consequences of physician dissatisfaction. Med Care 2006; 44: 234-242.

2 Kamien M. Staying in or leaving rural practice: 1996 outcomes of rural doctors' 1986 intentions. Med J Aust 1998; 169: 318-321.

3 Scott A, Gravelle H, Simoens S, et al. Job satisfaction and quitting intentions: a structural model of British general practitioners. $\mathrm{Br} J$ Industrial Relations 2006; 44: 519-540.

4 DeVoe J, Fryer GE, Straub A, et al. Congruent satisfaction: is there geographic correlation between patient and physician satisfaction? Med Care 2007; 45: 88-94.

5 Harris M, Proudfoot J, Jayasinghe UW, et al. Job satisfaction of staff and the team environment in Australian general practice. Med J Aust 2007; 186: 570-573.

6 McGlone SJ, Chenoweth IG. Job demands and control as predictors of occupational satisfaction in general practice. Med J Aust 2001; 175: 88-91.

7 Ulmer B, Harris M. Australian GPs are satisfied with their job: even more so in rural areas. Fam Pract 2002; 19: 300-303.

8 Walker KA, Pirotta M. What keeps Melbourne GPs satisfied in their jobs? Aust Fam Physician 2007; 36: 877-880.

9 Van Ham I, Verhoeven AAH, Groenier KH, et al. Job satisfaction among general practitioners: a systematic literature review. Eur J Gen Pract 2006; 12: 174-180.

10 Kluger MT, Townend K, Laidlaw T. Job satisfaction, stress and burnout in Australian specialist anaesthetists. Anaesthesia 2003; 58: 339-345.

11 Danesh-Meyer HV, Deva NC, Ku JY, et al. Differences in practice and personal profiles between male and female ophthalmologists. Clin Experiment Ophthalmol 2007; 35: 318-323.

12 Bruce CT, Sanger MM, Thomas PS, et al. Factors affecting female or male consultant stress in an Australian teaching hospital [letter]. Med $J$ Aust 2003; 179: 174-175.

13 Shehabi Y, Dobb G, Jenkins I, et al. Burnout syndrome among Australian intensivists: a survey. Crit Care Resusc 2008; 10: 312-315.

14 Crook HD, Taylor DM, Pallant JF, Cameron PA. Workplace factors leading to planned reduction of clinical work among emergency physicians. Emerg Med Australas 2004; 16: 28-34.

15 Bovier PA, Perneger TV. Predictors of work satisfaction among physicians. Eur J Public Health 2003; 13: 299-305.

16 Joyce CM, Scott A, Jeon SH, et al. The "Medicine in Australia: Balancing Employment and Life (MABEL)" longitudinal survey - protocol and baseline data for a prospective cohort study of Australian doctors' workforce participation. BMC Health Serv Res 2010; 10: 50.

17 Australasian Medical Publishing Company. AMPCo Direct Doctor Lists. http://www. ampcodirect.com.au (accessed May 2008).

18 Australian Institute of Health and Welfare. Medical labour force 2006. Canberra: AlHW, 2008. (AlHW Cat. No. HWL 42) http:// www.aihw.gov.au/publications/index.cfm/title/ 10620 (accessed Sep 2010).

19 Cooper CL, Rout U, Faragher B. Mental health, job satisfaction, and job stress among general practitioners. BMJ 1989; 298: 366-370.

20 Warr P, Cook J, Wall T. Scales for the measurement of some work attitudes and aspects of psychological well-being. J Occup Psychol 1979; 52: 129-148.

21 Kuehnle D, Scott A, Cheng T, et al. MABEL user manual: wave 1 release. Melbourne: University of Melbourne, 2010. https://mabel.org.au/ data/MABEL_User_Manual_Wave_1_De-identified.pdf (accessed Sep 2010).

22 Norton EC, Wang H, Ai C. Computing interaction effects and standard errors in logit and probit models. Stata J 2004; 4: 154-167.

23 An PG, Rabatin JS, Manwell LB, et al. Burden of difficult encounters in primary care: data from the Minimizing Error, Maximizing Outcomes Study. Arch Intern Med 2009; 169: 410-414.

24 Mathers N, Jones N, Hannay D. Heartsink patients: a study of their general practitioners. Br J Gen Pract 1995; 45: 293-296.

25 Hays R, Wynd S, Veitch C, et al. Getting the balance right? GPs who chose to stay in rural practice. Aust J Rural Health 2003; 11: 193-198.

26 Alexander C, Fraser J. Education, training and support needs of Australian trained doctors and international medical graduates in Australia: a case of special needs? Rural Remote Health 2007; 7: 681

27 Wilks CM, Oakley Browne M, Jenner BL. Attracting psychiatrists to a rural area -10 years on. Rural Remote Health 2008; 8: 824.

28 Eley D, Baker P. Does recruitment lead to retention? Rural clinical school training experiences and subsequent intern choices. Rural Remote Health 2006; 6: 511.

29 Henry JA, Edwards BJ, Crotty B. Why do medical graduates choose rural careers? Rural Remote Health 2009; 9: 1083.

(Received 20 Dec 2009, accepted 7 Sep 2010) 


\section{University Library}

\section{- M M N E R VA A gateway to Melbourne's research publications}

Minerva Access is the Institutional Repository of The University of Melbourne

Author/s:

Joyce, CM;Schurer, S;Scott, A;Humphreys, J;Kalb, G

Title:

Australian doctors' satisfaction with their work: results from the MABEL longitudinal survey of doctors

Date:

2011-01-03

\section{Citation:}

Joyce, C. M., Schurer, S., Scott, A., Humphreys, J. \& Kalb, G. (2011). Australian doctors' satisfaction with their work: results from the MABEL longitudinal survey of doctors.

MEDICAL JOURNAL OF AUSTRALIA, 194 (1), pp.30-33. https://doi.org/10.5694/ j.1326-5377.2011.tb04142.x.

Persistent Link:

http://hdl.handle.net/11343/91738 